Revista de

Contabilidade e

Organizações

www.rco.usp.br
DOI: http://dx.doi.org/10.11606/issn.1982-6486.rco.2020.166547
Journal of

Accounting and

Organizations

\title{
Personalidade e decisão financeira: um estudo com jovens do Exército Brasileiro
}

\author{
Personality traits and financial decisions: a study of youths in the Brazilian Army
}

João Rafael Martelo ${ }^{\mathrm{a}}$, Eveline Favero ${ }^{\mathrm{b}}$, Wagner Dantas de Souza Junior ${ }^{\mathrm{c}}$

${ }^{a}$ Universidade do Oeste do Paraná

"Universidade Estadual do Paraná

c Fundação Universidade Regional de Blumenau

Palavras-chave

Finanças comportamentais.

Escolhas intertemporais.

Traços de personalidade.

Valores humanos.

Exército Brasileiro.
Keywords

Behavioral finance.

Intertemporal choices.

Personality traits.

Human values.

Brazilian Army.
Informações do artigo

Recebido: 13 de fevereiro de 2020

Aprovado: 16 de junho de 2020

Publicado: 04 de agosto de 2020

\section{Resumo}

Este estudo analisa a relação entre traços de personalidade, valores humanos e características socioeconômicas nas escolhas intertemporais financeiras de jovens do Exército Brasileiro. Por meio de levantamento, participaram 218 jovens do serviço militar obrigatório de Cascavel-PR. Os resultados sugerem que, na média, jovens adultos demonstram maior paciência à impaciência financeira em suas escolhas intertemporais financeiras. Muitos jovens não apresentaram modelos de desconto intertemporal. A correlação de Pearson revelou associação significativa entre traços de personalidade de abertura, conscienciosidade e extroversão; valores humanos básicos como de realização, suprapessoal, existencial e interacional; e características socioeconômicas como o grau de escolaridade da mãe, estado civil, renda familiar, ambiente de criação e tamanho do município de origem. Estes resultados sugerem que fatores emocionais ou contextuais afetam as escolhas intertemporais financeiras de jovens adultos e podem influenciar por heurísticas afetivas o processamento de informações e a tomada de decisão individual.

\begin{abstract}
This study analyzes the relationships between personality traits, human values and socio-economic characteristics and intertemporal financial choices among young soldiers in the Brazilian army. Through the use of a survey, 218 young men in compulsory military service between 18 and 23 years of age from Cascavel, PR participated in our study. The results of our analyses suggest greater patience than impatience in their intertemporal financial choices, in a constant, non-hyperbolic discount model. Many young people did not present intertemporal discount models. The Pearson correlation coefficient revealed a significant association between personality traits of openness, conscientiousness and extroversion; basic human promotion, suprapersonal, existence and interaction values; and socioeconomic characteristics such as the mother's level of education, marital status, family income, growing up in a rural or urban environment and the size of the municipality of origin. These results suggest which emotional or contextual factors affect young people's intertemporal financial choices and how they can influence information processing and individual decision making through affective heuristics.
\end{abstract}

\section{Implicações práticas}

Para o campo de finanças comportamentais é possível sugerir traços da personalidade humana e do contexto sociocultural dos indivíduos, que afetam o processamento de informações e a tomada de decisões financeiras em jovens adultos. Estas variáveis influenciam a heurística afetiva, que por consequência, podem gerar vieses comportamentais. Dessa forma, modelos de análise da tomada de decisão devem considerar fatores psicológicos na análise do homem econômico.

Copyright (C) 2020 FEA-RP/USP. Todos os direitos reservados

Autor correspondente: Tel. (41) 3281-7328

E-mail: jrmnetword@hotmail.com (J. R. Martelo); evelinefavero@yahoo.com.br (E. Favero); wagnerdantas9@gmail.com (W. D. de Souza Junior)

Universidade Estadual do Paraná, Reitoria. Avenida Prefeito Lothario Meissner, 350 - Jardim Botânico, Curitiba/PR - 80210170, Brasil. 


\section{INTRODUÇÃO}

O dinheiro representa um comando de bens e serviços necessários para promover o interesse próprio do indivíduo em uma economia de livre iniciativa. Cada ser humano possui uma maneira diferente de lidar com o dinheiro e há evidências de que fatores da personalidade (Cadena \& Keys, 2015; Xu, Beller, Roberts, \& Brown, 2015; Wong \& Carducci, 2016) e características socioeconômicas (Coley \& Burgess, 2003; Pompian \& Longo, 2004; Kirby, Winston, \& Santiesteban; Bakker, 2017) possuem relação com as escolhas financeiras.

As finanças comportamentais estudam, desde Tversky e Kahneman (1974, 1979) e Kahneman (2002), vieses comportamentais que afetam as escolhas do homem econômico, sob a premissa da racionalidade limitada. Os estudos nessa área concentram-se nas características cognitivas que afetam o processamento de informações, porém assumem que heurísticas afetivas ou emoções também afetam este contexto.

Algumas questões ainda não foram respondidas ou consensuadas neste campo de estudo, tais como: quais fatores da personalidade afetam o julgamento dos indivíduos no processo de tomada de decisão? Como as emoções e heurísticas afetivas influenciam o processamento de informações? Como as características socioeconômicas, cultura e os valores individuais se relacionam nesse contexto? Há um esforço constante de diversos autores buscando compreender estas e outras questões para o avanço nas pesquisas na área (Norvilitis, Merwin, Osberg, Roehling, Young, \& Kamas, 2006; Brown \& Taylor, 2014; Potrich, Vieira, Campara, \& Santos, 2015; Campara, Vieira, \& Ceretta, 2016; Pacheco, Campara, \& Costa Jr., 2018).

Brown e Taylor (2014), por exemplo, afirmam que traços de personalidade podem interferir na dinâmica de decisões individuais de cunho econômico, podendo implicar inclusive em situações de endividamento familiar. Pacheco et al. (2018) complementam sugerindo que existem perfis psicológicos que são mais ou menos suscetíveis a problemas financeiros.

Esta situação pode se agravar ainda mais em jovens que iniciam a vida adulta, não são alfabetizados financeiramente e devem possuir responsabilidade sobre suas finanças. Norvilitis et al. (2006) relatam que jovens endividados possuem maior nível de estresse e mal-estar financeiro, o que pode prejudicar o seu futuro.

Com base no exposto, este estudo pretende identificar quais características da personalidade de jovens adultos possuem relação com suas escolhas intertemporais financeiras, pois tais características afetam a percepção, julgamento e comportamento individual em relação às decisões de consumo e investimento.

Nos diferenciamos dos estudos anteriores ao pesquisar a temática em jovens do sexo masculino que prestaram o serviço militar obrigatório, na faixa etária média de 19 anos. Este grupo de indivíduos está em início da idade adulta, em sua maioria não possui experiência com finanças e não possui viés de formação superior. Contudo, estes jovens incorporam valores institucionais do exército que podem influenciar suas escolhas intertemporais financeiras. Dessa forma, questiona-se: qual é o grau de associação entre traços da personalidade, valores humanos e características socioeconômicas nas escolhas intertemporais financeiras em jovens militares?

Para a análise das escolhas intertemporais, utilizou-se o modelo de desconto hiperbólico de Sutter, Kocher, Glätzle-Rützler e Trautmann (2013), que mede o nível de paciência ou impaciência financeira em escolhas financeiras, considerando as variáveis dinheiro, tempo e juros.

A análise da personalidade envolveu seis dimensões de personalidade do modelo HEXACO de Ashton e Lee (2007): honestidade-humildade, abertura, conscienciosidade, afabilidade, extroversão e estabilidade. Adicionamos o modelo de valores humanos básicos de Gouveia (2003), que analisa os valores experimentação, realização, suprapessoal, existencial, interacional e normativo do homem. Esta relação ainda não foi pesquisada no cenário brasileiro. Além disso, consideramos em nossas análises, características socioeconômicas como fatores influentes nas decisões financeiras, tais como o grau de escolaridade do indivíduo e de seus pais, estado civil, capacidade de poupança, gasto médio mensal, renda familiar, ambiente e tamanho da cidade de criação.

Os resultados evidenciam uma associação negativa entre os traços de personalidade de conscienciosidade e extroversão com as escolhas intertemporais dos jovens estudados. Isto sugere que características como autocontrole, organização e a expressividade podem afetar as escolhas financeiras nesta fase da vida adulta. Os valores humanos do existencialismo e da interação se associam positivamente com as decisões financeiras. Ainda, nota-se que o estado civil e nível de renda também afetam as decisões intertemporais. Outros resultados podem ser visualizados ao longo do trabalho.

Acredita-se que a compreensão dos fatores que moldam as decisões financeiras dos jovens nesta fase da vida adulta pode ser determinante no seu futuro, principalmente para a gestão do orçamento familiar. Os resultados desta pesquisa contribuem com novas evidências empíricas no campo de estudo de finanças comportamentais e para formuladores de políticas públicas no campo da educação financeira para jovens, sejam eles programas no ensino básico, fundamental, técnico, superior ou militar. 


\section{REVISÃO DA LITERATURA}

\subsection{Escolhas intertemporais financeiras, traços de personalidade e valores humanos}

Escolhas intertemporais são preferências individuais, que possibilitam trocas entre custos e benefícios a serem obtidos em distintos pontos temporais (Pimentel, Gonçalves, Scholten, Carvalho, \& Correia, 2012). Decisões de consumo, investimento, poupança ou planejamento de aposentadoria são alguns dos exemplos onde se apresenta a importância dos modelos de decisão das escolhas intertemporais no campo das finanças comportamentais (Muramatsu \& Fonseca, 2009).

O desenvolvimento de trabalhos nessa área ganhou relevância nas últimas décadas após as críticas de Tversky e Kahneman $(1974,1979)$ à Teoria Econômica da Utilidade Esperada e com a criação da Teoria do Prospecto. Neste campo, descobriu-se que vieses comportamentais e heurísticas cognitivas afetam o processamento de informações e a tomada de decisões do homem. Contudo, Kahneman (2002) afirma que outros fatores como as heurísticas afetivas também afetam o julgamento humano e devem ser considerados em modelos de tomada de decisão individual.

Desta forma, o estudo da personalidade, que é o campo afetivo ou emocional do homem, pode auxiliar no avanço dos estudos sobre fatores que influenciam o comportamento humano mediante a tomada de decisão. De acordo com Roberts (2009) os traços de personalidade são uma espécie de padrões de relativa duração de pensamentos, comportamentos e sentimentos que transparecem a tendência que o indivíduo possui ao responder a determinadas situações, sendo fundamentais para a essência da personalidade da pessoa.

Os traços de personalidade podem ser influenciados por características comportamentais, de atitude, motivação ou afetividade, entretanto possuem predisposição para manter-se estável na maneira de comportamento e de pensar do homem. Quanto às características atinentes a personalidade, essas têm se tornadas boas preditoras do comportamento humano, no que diz respeito à troca entre vantagens no presente em detrimento de ganhos futuros (De Faveri, 2017).

Ashton e Lee (2007) desenvolveram uma ferramenta para avaliar as dimensões da personalidade denominada Brief HEXACO Inventory, que corresponde a uma devolução do modelo Big Five criado na década de 1980 (Brown \& Taylor, 2014). Esse modelo inclui as dimensões honestidade-humildade, emotividade, extroversão, afabilidade, conscienciosidade e abertura à experiência. As seis dimensões podem ser mais bem compreendidas a partir das definições apresentadas por De Faveri (2017).

Fatores da personalidade relacionam-se com decisões financeiras na medida em que influenciam o processamento de informações e julgamento da tomada de decisão. Por exemplo, pessoas com níveis de impaciência financeira apresentam traços da personalidade associados à impulsividade, dificuldade de autocontrole, neurose, dentre outros fatores, que acabam por afetar a sua tomada de decisão (Cadena \& Keys, 2015).

Borghans, Duckworth, Heckman e Ter Weel (2008) avaliaram sistemas que tratam de medidas de traços de personalidade, associando-os com parâmetros de preferência mais utilizados pelos economistas. Concluíram que as medidas de capacidade cognitiva sofrem influência dos traços de personalidade, sendo possível conceituar e medir esses traços, sendo ainda preditores de resultados econômicos e sociais.

Shamosh e Gray (2008) evidenciaram em sua pesquisa que pessoas com maior nível de inteligência possuem uma menor propensão por recompensas antecipadas, demonstrando assim um menor índice de impaciência em suas relações com as finanças. Por fim, Xu et al. (2015) encontraram evidências que jovens adultos mais conscientes, por saberem lidar com a impulsividade, têm menor probabilidade de sofrerem dificuldades financeiras, enquanto os mais neuróticos são mais prováveis de sofrer com as finanças. Desta maneira, os estudos sugerem que há uma relação entre traços de personalidade e o comportamento do homem frente às finanças.

Norvilitis et al. (2006) pesquisaram o alto nível de endividamento no cartão de crédito de estudantes universitários americanos. Fatores como conhecimento financeiro, idade, número de cartões, atraso no recebimento de salário e atitudes em relação ao uso do cartão estavam relacionados à dívida. Os estudantes que reportaram maiores dívidas relataram maior estresse e menor bem-estar financeiro. Os autores enquadraram os fatores que colocam o indivíduo em risco de desenvolver problemas com dívidas no cartão de crédito em: conhecimento e atitude financeira, fatores da personalidade e características socioeconômicas. 
Sadi, Asl, Rostami, Gholipour e Gholipour (2011) expressaram a relação entre personalidade e decisões financeiras com uma amostra de 200 especialistas de fundos de investimentos na Índia. Os autores encontraram uma forte relação entre personalidade e os erros de percepção (vieses comportamentais) dos respondentes, indicando que traços da personalidade influenciam as decisões dos indivíduos.

Brown e Taylor (2014) concluíram que os traços de personalidade de abertura e extroversão apresentam influência significativa tanto nas aplicações financeiras quanto nas dívidas das famílias. O traço de extroversão revelou um efeito adverso no volume de ativos financeiros. Outra descoberta relevante foi a verificação do forte indício da interferência dos traços de personalidade na dinâmica de tomada de decisão financeira individual.

No Brasil, Pacheco et al. (2018), demonstraram a predominância dos traços de personalidade conscienciosidade e abertura à experiência em servidores públicos de uma universidade no sul do país, além de indicarem elevado conhecimento financeiro e baixa atitude ao endividamento. Este perfil sugere ser menos suscetível a problemas financeiros dentre os demais servidores pesquisados.

Por fim, os valores humanos são fundamentos éticos que influenciam a conduta humana e são importantes preditores de atitudes, sentimentos e comportamentos, podendo ter reflexos em atributos culturais, sociais e econômicos (Queiroga, Gouveia, Penha, Pessoa, \& Meira, 2006).

Segundo Roccas e Sagiv (2010) ao considerar um sistema de valorização, os valores humanos básicos podem ser dispostos em três níveis de orientação: valores pessoais (experimentação e realização), valores centrais (existência e suprapessoal) e valores sociais (normativo e interacional). Gouveia (2003) desenvolveu um instrumento que avalia os valores humanos básicos no Brasil, denominado Questionário de Valores Básicos.

Valores pessoais de experimentação e realização podem influenciar a racionalidade na tomada de decisão financeira, levando os indivíduos a tomarem decisões baseadas em estímulos ou desejos subjetivos, o que seriam consideradas decisões financeiras não ótimas. Já valores centrais, como o existencialismo e suprapessoal, podem manter o homem econômico racional a preferir escolhas intertemporais que maximize a sua riqueza particular (Gouveia, 2003). Assim, acredita-se existir algum nível de associação entre os valores humanos básicos e as escolhas intertemporais financeiras dos agentes econômicos.

Para dar continuidade às pesquisas, pretende-se compreender a relação entre traços de personalidade e escolhas intertemporais financeiras em jovens em início da idade adulta. Para complementar a análise, buscamos relacionar além das dimensões da personalidade, os valores humanos e as características socioeconômicas dos indivíduos.

\subsection{Características socioeconômicas e decisões financeiras}

As características socioeconômicas dos indivíduos também possuem relação com escolhas intertemporais financeiras. Bakker (2017) afirma que pessoas com menor renda, em comparação com as pessoas mais ricas, preocupam-se mais com suas necessidades mais imediatas e, portanto, descontam mais o futuro, priorizando ações financeiras de curto prazo.

Pompian e Longo (2004), ao estudar o público de investidores, constataram que traços de personalidade e o sexo do indivíduo propiciam diferentes resultados nos vieses de investimentos. Complementando estes estudos, Kirby et al. (2005) evidenciaram que a impaciência financeira está relacionada com o nível de escolaridade dos indivíduos.

No Brasil, Potrich et al. (2015) investigaram a relação entre o nível de educação financeira e as características socioeconômicas e demográficas de habitantes do Rio Grande do Sul. Os resultados apontam maiores níveis de educação financeira entre os homens, solteiros, que não possuem dependentes, estudantes, com um maior nível de escolaridade, tanto seu, quanto dos seus pais, com maiores faixas de renda própria e familiar.

Campara et al. (2018) estudaram a relação entre características socioeconômicas e a atitude frente ao endividamento de indivíduos do Rio Grande do Sul no Brasil. Com uma amostra composta por adultos em fase de maturidade, com idade média de 43 anos, os autores alegam que os entrevistados não possuíam tendência ao acúmulo de dívida, mas sim uma baixa atitude ao endividamento, não tolerantes ao risco, não compradores compulsivos e são caracterizados por bom comportamento financeiro. Segundo os achados, os homens devem preocupar-se de maneira mais efetiva com a manutenção de uma boa atitude financeira, evitar compras compulsivas e manter um comportamento financeiro adequado para a sobrevivência familiar. 
Desta forma, visualizamos que, de modo geral, as características socioeconômicas em conjunto com traços da personalidade influenciam as escolhas intertemporais financeiras dos indivíduos e influenciam o seu comportamento perante o dinheiro. Assim, torna-se promissor verificar empiricamente quais fatores são indutores desse comportamento em jovens adultos no Brasil.

\section{PROCEDIMENTOS METODOLÓGICOS}

Esta pesquisa pode ser classificada como quantitativa, exploratória e por levantamento. Os dados foram coletados no início de 2019. A população-alvo escolhida foram jovens que incorporaram o serviço militar obrigatório no ano de 2018/2019 na cidade de Cascavel, no Paraná. A população de recrutas que esteve servindo no período perfaz um total de 470 militares, de um total de 10.500 jovens com 18 e 19 anos no município, sendo metade aproximadamente do sexo masculino, segundo o último Censo do IBGE. Estes jovens representam cerca de 5\% da população da mesma faixa etária e sexo do município.

Do total de 227 questionários respondidos, 9 tiveram que ser descartados por preenchimento incorreto, aproveitando-se 218 questionários. Este número atende o nível exigido pela amostragem estatística, considerando um erro amostral de 5\% e um nível de $95 \%$ de confiança dos dados.

O questionário desenvolvido conteve quatro seções, com o intuito de medir o nível de impaciência financeira em relação às escolhas intertemporais, dimensões da personalidade, valores humanos e as características socioeconômicas dos jovens militares.

A primeira seção do instrumento conteve um teste de verificação da impaciência ou paciência nas escolhas intertemporais financeiras, por meio do modelo de desconto hiperbólico de Sutter et al. (2013), traduzido e adaptado por De Faveri (2017).

Neste teste os participantes escolhem entre determinadas recompensas em dois momentos diferentes no tempo: uma recompensa imediata e outra recompensa maior no futuro. Ao todo foram utilizadas 8 listas de escolhas que continham 10 itens em cada, nas quais a recompensa antecipada permaneceu inalterada no tempo e a recompensa futura era ampliada monotonamente ao longo do tempo.

Para analisar os resultados dos níveis de impaciência financeira da amostra, calculou-se o equivalente futuro de cada uma das listas e comparou-se duas a duas. Se o valor médio do equivalente futuro da Lista 1 for maior que o da lista 2, considera-se que o indivíduo desconta o tempo hiperbolicamente, pois ele se comporta de forma impaciente no curto prazo e paciente no longo prazo. Caso contrário, ele apresenta um viés de desconto constante. Para complementar a análise, foi realizado um teste t de médias para verificar se os resultados são significativos estatisticamente.

A segunda seção abrangeu o teste de verificação de personalidade Brief HEXACO Inventory, desenvolvido por De Vries (2013), traduzido por De Faveri (2017). O teste é composto de 24 afirmativas distribuídos em uma escala Likert de 1 (discordo muito) a 5 (concordo muito). A terceira seção envolveu o Questionário de Valores Básicos desenvolvido por Gouveia (2003). Esse teste possui 18 afirmações e uma escala Likert de 7 pontos. Para a execução desta pesquisa, a escala foi adaptada para 5 pontos.

A quarta seção do instrumento abordou as características socioeconômicas dos indivíduos. As variáveis analisadas foram a escolaridade do respondente e de seus pais, estado civil, controle financeiro, renda familiar, ambiente de criação (área rural ou urbana) e tamanho do município de origem. O instrumento da pesquisa é apresentado na Figura 1.

Para analisar a o grau de associação e a direção entre as escolhas intertemporais financeiras, os traços de personalidade, valores humanos e características socioeconômicas, optou-se pela análise de correlação de Pearson.

Para a análise de correlação, transformaram-se as variáveis socioeconômicas ordinais em variáveis dummy, sendo: se a escolaridade do mãe for maior que a do filho, valor igual 1, se não igual a 0 ; se a escolaridade do pai for maior que a do filho, valor igual a 1 , se não igual a 0 . Se o jovem possuir união estável ou for casado, valor igual a 1 , se não igual a 0 ; se poupa algum valor do salário, valor igual a 1 , se não igual a 0 ; Se gastar mais que 2/3 do salário com gastos não essenciais, valor igual a 1 , se não igual a 0 ; a se renda familiar for até 2 salários mínimos, valor igual a 1 , se não igual a 0 . Se o jovem foi criado em área rural, valor igual a 1 , se não igual 0 ; se a cidade em que foi criado for considerada pequena (até 100.000 habitantes), valor igual a 1, se não igual a 0 . 


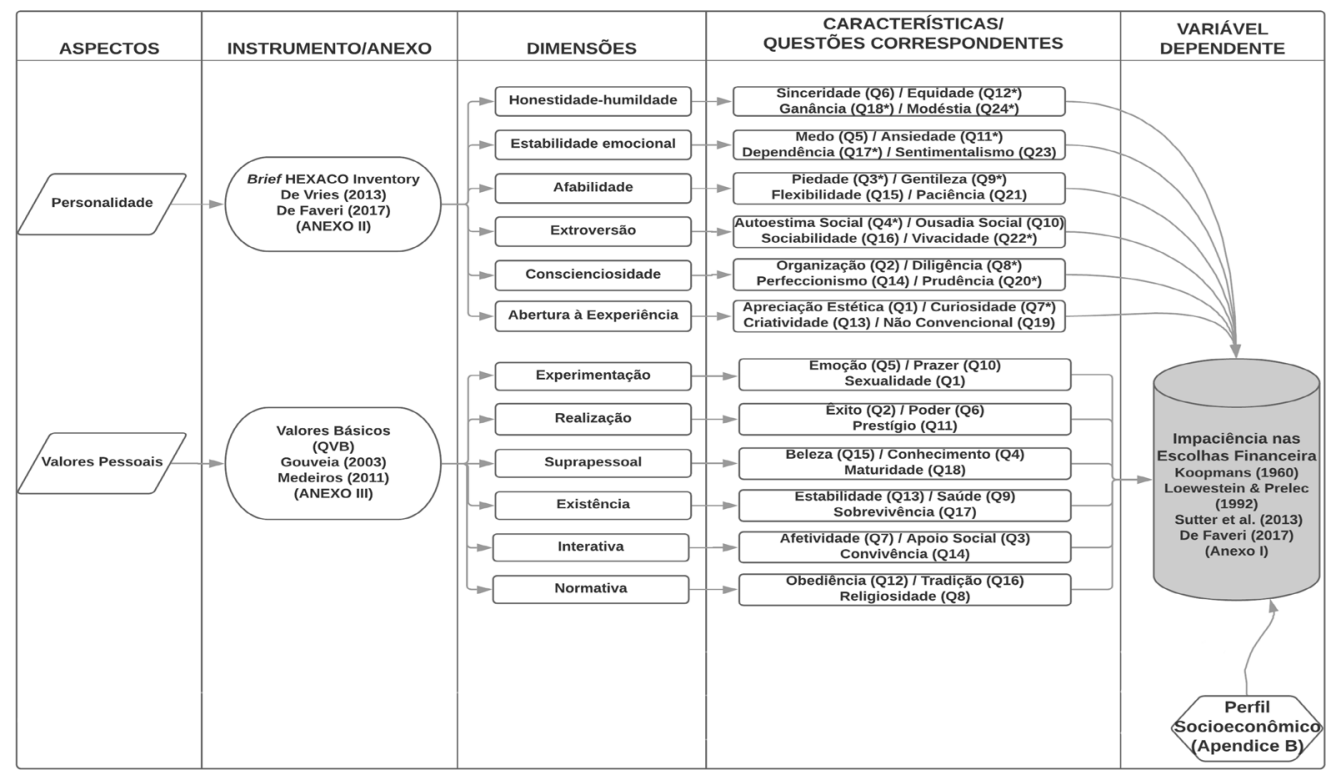

Figura 1. Dimensões de análise do instrumento de coleta de dados da pesquisa Fonte: Adaptado pelos autores de Gouveia (2003), Sutter et al. (2013) e De Faveri (2017).

\section{DESCRIÇÃO E ANÁLISE DOS RESULTADOS}

Em resumo, os jovens militares da amostra possuem em média 19 anos, solteiros, com o ensino médio incompleto e baixa instrução dos familiares. Cerca de $38 \%$ dos jovens afirmaram nunca conseguir poupar dinheiro e $43 \%$ conseguem poupar até $\mathrm{R} \$ 300,00$. Ainda, $74 \%$ comprometem mais de $40 \%$ de sua renda com gastos de lazer e ativos. Em relação a renda familiar média, $27 \%$ dos jovens recebem até 2 salários mínimos e $37 \%$ recebem de $\mathrm{R} \$ 2.001,00$ a $\mathrm{R} \$ 4.000,00$. Em relação a criação destes jovens, $65 \%$ foram criados em um ambiente urbano e $35 \%$ em ambiente rural. Por fim, $47 \%$ afirmam terem sido criados em uma cidade de pequeno porte e $53 \%$ em médio ou grande porte.

A Tabela 1 apresenta a estatística descritiva da dimensão dos traços de personalidade e dos valores humanos investigados na amostra. É possível notar que predominam traços da personalidade de afabilidade e conscienciosidade, observando o valor da nota de corte da média maior que a mediana. No entanto, nenhum valor se destacou em questão. No quesito valores humanos básicos, os jovens possuem maior concordância nos valores de existência, interatividade e normatividade.

Tabela 1. Estatística descritiva dos traços de personalidade e dos valores humanos da amostra

\begin{tabular}{llccc}
\hline & Descrição & Média & Mediana & Moda \\
\hline & Honestidade e Humildade & 14,4 & 15 & \\
& Estabilidade Emocional & 16,5 & 17 & \\
Modelo HEXACO & Afabilidade & 13,2 & 13 & \\
& Extroversão & 11,9 & 12 & \\
& Conscienciosidade & 15,4 & 15 & \\
& Abertura & 10,5 & 11 & 4 \\
\hline \multirow{5}{*}{ Questionário de Valores Básicos } & Experimentação & & 4,0 & 3,6 \\
& Realização & & 4,0 & 4,3 \\
& Suprapessoal & & 4,0 & 4,3 \\
& Existência & & 4,3 & 4,3 \\
\hline
\end{tabular}

Fonte: dados da pesquisa.

A Tabela 2 apresenta as escolhas intertemporais dos jovens militares frente às decisões financeiras. Em primeiro lugar, foi necessário segregar os jovens que responderam as listas em modelo de desconto constante, desconto hiperbólico ou sem desconto. 
O primeiro grupo decide de maneira racional na relação dinheiro, juros e tempo. O segundo não faz distinção entre as listas, sendo o seu resultado considerando não importar a relação entre estas variáveis. O terceiro grupo apresenta um modelo de desconto hiperbólico, isto é, jovens que preferem antecipar o recebimento de valores no presente ao receber este valor no futuro, mesmo que compense financeiramente.

Classificamos o primeiro grupo de pacientes e os últimos de impacientes financeiramente. Na comparação entre as listas 1 e 2, 12\% dos jovens apresentaram um modelo de desconto hiperbólico em suas decisões; nas listas 3 e 4, 20\% listas 5 e $6,10 \%$; listas 7 e $8,16 \%$. O que se percebe é que quanto maior o tempo de espera entre as decisões financeiras, mais estes jovens querem antecipar suas escolhas, apresentando algum nível de impaciência. No entanto, ao comparar as listas pelas suas médias, notamos que o modelo que predomina na média de grupos analisados é o modelo de desconto constante.

Tabela 2. Modelo de desconto hiperbólico das escolhas intertemporais financeiras

\begin{tabular}{|c|c|c|c|c|c|}
\hline \multicolumn{2}{|c|}{ Lista } & $\begin{array}{l}\text { Desconto } \\
\text { Constante }\end{array}$ & Sem Desconto & $\begin{array}{c}\text { Desconto } \\
\text { Hiperbólico }\end{array}$ & $\begin{array}{c}\text { Frequência Relativa do } \\
\text { Desconto Hiperbólico }\end{array}$ \\
\hline \multicolumn{2}{|c|}{$\mathrm{EF} 1>\mathrm{EF} 2$} & 135 & 57 & 26 & $12 \%$ \\
\hline \multicolumn{2}{|c|}{$\mathrm{EF} 3>\mathrm{EF} 4$} & 33 & 142 & 43 & $20 \%$ \\
\hline \multicolumn{2}{|c|}{ EF5 $>$ EF6 } & 130 & 67 & 21 & $10 \%$ \\
\hline \multicolumn{2}{|c|}{$\mathrm{EF} 7>\mathrm{EF} 8$} & 75 & 108 & 35 & $16 \%$ \\
\hline Lista & Amostra & Média & Comparação & Teste $\mathrm{t}$ & Conclusão \\
\hline EF1 & \multirow{2}{*}{161} & 4,84 & \multirow{2}{*}{$\mathrm{EF} 1<\mathrm{EF} 2$} & \multirow{2}{*}{$(9,90)^{*}$} & \multirow{2}{*}{ Desconto constante } \\
\hline EF2 & & 6,53 & & & \\
\hline EF3 & \multirow{2}{*}{76} & 7,55 & \multirow{2}{*}{$\mathrm{EF} 3>\mathrm{EF} 4$} & \multirow{2}{*}{1,6} & \multirow{2}{*}{ Desconto hiperbólico } \\
\hline EF4 & & 7,13 & & & \\
\hline EF5 & \multirow{2}{*}{151} & 3,04 & \multirow{2}{*}{$\mathrm{EF} 5<\mathrm{EF} 6$} & \multirow{2}{*}{$(9,93)^{*}$} & \multirow{2}{*}{ Desconto constante } \\
\hline EF6 & & 4,59 & & & \\
\hline EF7 & \multirow{2}{*}{110} & 6,37 & \multirow{2}{*}{$\mathrm{EF} 7<\mathrm{EF} 8$} & \multirow{2}{*}{$(2,14)^{*}$} & \multirow{2}{*}{ Desconto constante } \\
\hline EF8 & & 6,80 & & & \\
\hline
\end{tabular}

Fonte: dados da pesquisa.

Nota: *Significativo em até $5 \%$.

A Tabela 3 apresenta a correlação de Pearson entre os constructos analisados. Na categoria traços de personalidade, as dimensões abertura, conscienciosidade e extroversão apresentaram um grau de associação negativo e significativo com os níveis de paciência financeira. Assim, quanto maior o nível de equivalente futuro do modelo de desconto hiperbólico aplicado, menor é o valor das dimensões destes traços de personalidade.

$\mathrm{Na}$ categoria de valores humanos, observa-se uma relação negativa e significativa entre o nível de paciência financeira nas escolhas intertemporais e as dimensões de experimentação, realização e suprapessoal. De outro modo, as dimensões existencial e suprapessoal associam-se positiva e significativa com os equivalentes futuros.

Entre as variáveis socioeconômicas, nota-se uma associação negativa e significativa com a escolaridade da mãe, estado civil, poupança e renda familiar com os níveis de paciência financeira. Ao contrário, encontramos que o tipo e o tamanho do município de origem possuem um grau de associação positivo e significativo com os níveis de paciência financeira. 
Tabela 3. Correlação entre fatores dos traços de personalidade e a impaciência financeira

\begin{tabular}{|c|c|c|c|c|c|c|c|c|c|}
\hline Modelos & Variáveis & ef1 & ef 2 & ef3 & ef4 & ef5 & ef6 & ef7 & ef8 \\
\hline \multirow{6}{*}{$\begin{array}{c}\text { Modelo HEXACO } \\
\text { de Personalidade }\end{array}$} & Honestidade-humildade & 0,10 & 0,07 & 0,01 & 0,03 & 0,09 & 0,09 & 0,03 & 0,05 \\
\hline & Abertura & $-0,06$ & $-0,14 * *$ & $-0,17^{*}$ & $-0,14 * *$ & $-0,05$ & 0,02 & $-0,05$ & $-0,05$ \\
\hline & Conscienciosidade & $-0,18^{*}$ & $-0,24 *$ & $-0,08$ & 0,00 & $-0,06$ & $-0,01$ & $-0,18 *$ & $-0,13$ \\
\hline & Afabilidade & $-0,09$ & 0,03 & 0,03 & 0,11 & $-0,08$ & $-0,02$ & 0,04 & 0,05 \\
\hline & Extroversão & $-0,06$ & $-0,15^{* *}$ & $-0,11 * * *$ & $-0,08$ & $-0,12 * * *$ & $-0,06$ & $-0,18^{*}$ & $-0,07$ \\
\hline & Estabilidade & $-0,24$ & 0,03 & 0,02 & 0,04 & 0,01 & $-0,02$ & 0,04 & 0,06 \\
\hline \multirow{6}{*}{$\begin{array}{c}\text { Modelo de Valores } \\
\text { Humanos Básicos }\end{array}$} & Experimentação & $-0,05$ & $-0,06$ & $-0,12$ & $-0,09$ & $-0,15^{* *}$ & $-0,01$ & $-0,02$ & $-0,01$ \\
\hline & Realização & $-0,13 * * *$ & $-0,05$ & $-0,16^{* *}$ & $-0,11 * * *$ & $-0,11$ & $-0,10$ & $-0,11$ & $-0,09$ \\
\hline & Suprapessoal & $-0,10$ & $-0,16^{* *}$ & $-0,11 * * *$ & 0,08 & $-0,09$ & $-0,06$ & $-0,11 * * *$ & $-0,09$ \\
\hline & Existencial & $0,12 * * *$ & 0,08 & $0,12 * * *$ & $0,22 *$ & $-0,03$ & $-0,02$ & 0,10 & 0,03 \\
\hline & Interacional & $-0,03$ & $-0,04$ & 0,00 & $0,12 * * *$ & $-0,02$ & 0,00 & 0,04 & 0,07 \\
\hline & Normativo & 0,04 & 0,07 & $-0,07$ & 0,03 & $-0,07$ & 0,03 & 0,04 & 0,06 \\
\hline \multirow{8}{*}{$\begin{array}{c}\text { Caraterísticas } \\
\text { Socioeconômicas }\end{array}$} & Escolaridade mãe & $-0,05$ & $-0,06$ & 0,10 & $-0,04$ & $-0,01$ & $-0,13^{* *}$ & 0,00 & $-0,04$ \\
\hline & Escolaridade pai & 0,06 & 0,07 & 0,07 & $-0,02$ & 0,06 & $-0,04$ & 0,07 & $-0,03$ \\
\hline & Estado civil & $-0,15^{* *}$ & $-0,06$ & $-0,04$ & $-0,17^{*}$ & $-0,03$ & 0,02 & $-0,03$ & $-0,07$ \\
\hline & Poupança & $-0,10$ & $-0,09$ & $-0,10$ & $-0,06$ & 0,05 & $-0,12$ & $-0,16^{*}$ & $-0,11$ \\
\hline & Gasto Mensal & 0,09 & $-0,03$ & 0,04 & 0,08 & $-0,01$ & 0,06 & 0,04 & 0,06 \\
\hline & Renda & $-0,14 * *$ & $-0,04$ & 0,00 & 0,01 & 0,09 & 0,04 & $-0,06$ & $-0,08$ \\
\hline & Rural ou urbano & 0,03 & 0,00 & $0,15^{* *}$ & $0,15^{* *}$ & 0,04 & 0,00 & 0,03 & 0,03 \\
\hline & Tamanho da cidade & 0,11 & 0,10 & 0,06 & 0,08 & 0,06 & 0,06 & $0,16^{* *}$ & 0,09 \\
\hline
\end{tabular}

Fonte: dados da pesquisa.

Nota: P-valor significativo a: $1 \% *, 5 \% * *$ e $10 \% * * *$ 


\section{DISCUSSÃO DOS RESULTADOS}

Ao analisar a amostra do estudo, a relação entre impaciência financeira e os valores suprapessoais pode estar relacionado às experiências e desafios que os jovens precisam superar na vida, para suprir as suas necessidades de crescimento.

Existe uma relação inversa e significativa entre o nível de impaciência financeira e a extroversão. A extroversão é uma característica da personalidade de pessoas confiantes quando lideram grupos de pessoas, desfrutam de encontros e interações sociais, possuem sentimentos positivos de experiência, de entusiasmo e de energia (De Vries, 2013).

Avaliando esta perspectiva para a amostra da pesquisa, nota-se que os jovens militares mais extrovertidos tendem a ser mais impacientes e preferem escolhas intertemporais financeiras hoje em detrimento do longo prazo. Esta análise é sustentada pelo baixo ou nenhum grau de poupança e o alto nível de gasto médio mensal com gastos de lazer ou prestações que os indivíduos possuem. Porém, segundo Weller e Tikir (2011), é possível que ocorra resultados controversos sob essa hipótese.

Existe uma relação inversa e significativa entre o nível de impaciência financeira e a conscienciosidade. Uma alta característica de conscienciosidade está relacionada à organização e disciplina dos indivíduos. Este valor é amplamente disseminado na estrutura do Exército Brasileiro. Este resultado coaduna com Weller e Tikir (2011) e Xu et al. (2015).

Weller e Tikir (2011) constataram que indivíduos conscienciosos são geralmente mais cuidadosos, mais propensos a manter o foco na tarefa e, como tal, podem ser mais propensos a considere os riscos e benefícios de certas atividades que podem, em última análise, levar a um menor envolvimento em riscos desnecessários. Os autores observaram correlações negativas significativas da extroversão com a tomada de riscos e benefícios percebidos para os domínios da saúde, segurança e ética de sua amostra.

Ressalta-se que Xu et al. (2015) também investigaram se traços de personalidade explicam um conjunto de indicadores de dificuldades financeiras. Entre os resultados os autores descobriram que adultos jovens mais conscientes têm menor probabilidade de sofrer dificuldades financeiras, enquanto mais neuróticos são mais prováveis.

Existe uma relação negativa e significativa entre o nível de impaciência financeira e a capacidade de poupar do indivíduo. Este resultado comparou os indivíduos que não conseguem poupar nada frente aos que conseguem poupar alguma quantia monetária, sendo que 38\% dos jovens entrevistados declararam que não conseguem poupar nada. Loewenstein et al. (2003) haviam afirmado que indivíduos que apresentam dificuldades na retenção de conhecimento em entender e imaginar situações futuras não consideram importantes os acontecimentos que ocorrerão no longo prazo. Por consequência, eles implicam em taxas de desconto maiores que afetam o seu nível de poupança para a aposentadoria.

Existe uma relação positiva e significativa entre o nível de impaciência financeira e a renda familiar. Nesta análise, verificou-se que os indivíduos que possuem uma baixa renda são mais impacientes financeiramente. Bakker (2017) já havia verificado em sua pesquisa que, indivíduos com menor renda em relação a indivíduos com maior renda, preocupam-se com necessidades mais imediatas e por isso descontam mais o futuro, priorizando ações financeiras de curto prazo.

Ainda, existe uma relação positiva e significativa entre o nível de impaciência financeira e o tamanho e o grau de urbanização do município. Indivíduos que consideram ser criados em uma cidade pequena, de até 100 mil habitantes, e em área urbana, tendem a ser mais impacientes financeiramente.

Por fim, os fatores da personalidade, valores básicos e características socioeconômicas tais como a vida precoce, estrutura familiar, saúde e educação, entre outros, são o pano de fundo para gerar esse comportamento decisório (Xu et al., 2015). Assim, em síntese, esta pesquisa conseguiu atingir o seu objetivo, ao apresentar a relação entre alguns dos traços da personalidade humana e níveis de impaciência financeira.

\section{CONCLUSÕES}

O objetivo desta pesquisa foi analisar a relação entre os traços de personalidade como personalidade, valores humanos e as características socioeconômicas com a impaciência nas escolhas intertemporais financeiras em jovens militares do Exército Brasileiro. 
Os resultados sugerem que os indivíduos utilizam modelos de desconto constante ao invés de hiperbólico. A análise de correlação sugeriu a associação entre algumas das dimensões da personalidade, valores humanos e características socioeconômicas e as escolhas intertemporais individuais.

A escolha intertemporal é um determinante central em relação às decisões econômicas, que vão desde a educação, a poupança e investimento. Porém, muito, além disso, elas moldam o comportamento do agente, o que pode auxiliar a formação dos soldados dentro da própria instituição do Exército Brasileiro, muito além da atividade fim. Recrutas com menor impaciência financeira tendem a se tornar Soldados, Cabos, Sargentos ou Oficiais no futuro que tomam decisões considerando um raciocínio estratégico por trás, avaliando a relação risco-retorno das alternativas que lhe são postas e tendo uma postura organizada, planejadora, paciente e menos impetuosa.

Como limitação da pesquisa é possível citar os modelos de avaliação dos traços de personalidade escolhidos pelos pesquisadores. Para pesquisas futuras recomenda-se adotar outros instrumentos para mensurar impaciência financeira, considerando recompensas financeiras e não financeiras e analisar os vieses relacionados ao desconto hiperbólico com produtos financeiros oferecidos por instituições financeiras. Espera-se que esta pesquisa estimule novos estudos sobre finanças comportamentais.

\section{REFERÊNCIAS}

Ashton, M. C., \& Lee, K. (2007). Empirical, theoretical, and practical advantages of the HEXACO model of personality structure. Personality and Social Psychology Review, 2(11), p.150-166. DOI: https://doi. org/10.1177\%2F1088868306294907.

Bakker, B. N. (2017). Personality traits, income, and economic ideology. Political Psychology, 38(6), p. 10251041. DOI: https://doi.org/10.1111/pops.12349.

Borghans, L., Duckworth, A. L., Heckman, J. J., \& Ter Weel, B. (2008). The economics and psychology of personality traits. Journal of Human Resources, 43(4), p. 972-1059.

Brown, S., \& Taylor, K. (2014). Household finances and the 'Big Five' personality traits. Journal of Economic Psychology, 45, p. 197-212. DOI: https://doi.org/10.1016/j.joep.2014.10.006.

Cadena, B. C., \& Keys, B. J. (2015). Human capital and the lifetime costs of impatience. American Economic Journal: Economic Policy, 7(3), p. 126-53. DOI: http://dx.doi.org/10.15185/izawol.233.

Campara, J. P., Vieira, K. M., \& Ceretta, P. S. (2016). Entendendo a atitude ao endividamento: fatores comportamentais e variáveis socioeconômicas o determinam? Revista Eletrônica de Ciência Administrativa, 15(1), p. 5-24. DOI: 10.21529/RECADM. 2016002.

De Faveri, D. B. (2017). Impaciência nas escolhas intertemporais: uma abordagem comportamental. Tese de Doutorado em Economia, Universidade Federal de Santa Catarina, Florianópolis, SC, Brasil.

De Faveri, D. B., \& Knupp, P. S. (2018). Behavioral finance: the relationship between personality traces and behavioral biases. BASE - Revista de Administração e Contabilidade da Unisinos, 15(1), p. 18-30. DOI: doi: 10.4013/base.2018.151.02.

De Vries, R. E. (2013). The 24-item Brief HEXACO Inventory (BHI). Journal of Research in Personality, 47(6), p. 871-880. DOI: https://doi.org/10.1016/j.jrp.2013.09.003.

Gouveia, V. V. (2003). A natureza motivacional dos valores humanos: evidências acerca de uma nova tipologia. Estudos de Psicologia, 8(3), p. 431-443. DOI: https://doi.org/10.1590/S1413-294X2003000300010.

Kahneman, D. (2002). Maps of bounded rationality: A perspective on intuitive judgment and choice. Nobel prize lecture, $8,351-401$.

Kirby, K. N., Winston, G. C., \& Santiesteban, M. (2005). Impatience and grades: delay-discount rates correlate negatively with college GPA. Learning and Individual Differences, 15(3), p. 213-222. DOI: doi/10.1016/j. lindif.2005.01.003.

Loewenstein, G., O’Donoghue, T., \& Rabin, M. (2003). Projection bias in the predicting of future utility. Quarterly Journal of Economics, 118(4), p. 1209-1248. DOI: https://doi.org/10.1162/003355303322552784.

Muramatsu, R., \& Fonseca, P. (2009). Economia e psicologia na explicação da escolha intertemporal. Revista de Economia Mackenzie, 6(1), p. 87-112. 
Norvilitis, J. M., Merwin, M. M., Osberg, T. M., Roehling, P. V., Young, P., \& Kamas, M. M. (2006). Personality factors, money attitudes, financial knowledge, and credit-card debt in college students. Journal of Applied Social Psychology, p. 36(6), 1395-1413. DOI: https://doi.org/10.1111/j.0021-9029.2006.00065.x.

Pacheco, G. B., Campara, J. P., \& Costa Jr., N. C. A. (2018). Traços de personalidade, atitude ao endividamento e conhecimento financeiro: um retrato dos servidores da Universidade Federal de Santa Catarina. Revista de Ciências da Administração, 1(3), p. 54-73. DOI: https://doi.org/10.5007/2175-8077.2018V20n52p54.

Pimentel, D., Gonçalves, G., Scholten, M., Carvalho, P. L. M. D., \& Correia, M. F. (2012). Efeitos contextuais na escolha intertemporal: evidência contra modelos de desconto. Análise Psicológica, 30(3), p. 269-284.

Pompian, M. M, \& Longo, J. M. (2004). A new paradigm for practical application of behavioral finance: creating investment programs based on personality type and gender to produce better investment outcomes. The Journal of Wealth Management, 7(2), p. 9-15. DOI: https://doi.org/10.3905/jwm.2004.434561.

Potrich, A. C. G., Vieira, K. M., Campara, J. P., \& Santos, L. F. D. O. (2015). Educação Financeira dos Gaúchos: Proposição de uma Medida e Relação com as Variáveis Socioeconômicas e Demográficas. Sociedade, Contabilidade e Gestão, 9(3), p. 109-129. DOI: https://doi.org/10.21446/scg_ufrj.v9i3.13335.

Queiroga, F., Gouveia, V. V., Penha, L. C. M., Pessoa, V., \& Meira, M. (2006). Intenção de comportamento socialmente responsável do consumidor: sua relação com os valores humanos básicos. Psico, 11(2), p. 239248. DOI: https://doi.org/10.1590/S1413-82712006000200012.

Roberts, B. W. (2009). Back to the future: Personality and assessment and personality development. Journal of Research in Personality, 43(2), p. 137-145. DOI: https://dx.doi.org/10.1016\%2Fj.jrp.2008.12.015.

Roccas, S., \& Sagiv, L. (2010). Personal values and behavior: taking the cultural context into account. Social and Personality Psychology, 4(1), p. 30-41. DOI: https://doi.org/10.1111/j.1751-9004.2009.00234.x.

Sadi, R., Asl, H. G., Rostami, M. R., Gholipour, A., \& Gholipour, F. (2011). Behavioral finance: the explanation of investors' personality and perceptual biases effects on financial decisions. International Journal of Economics and Finance, 3(5), p. 234-241. DOI: https://doi.org/10.5539/ijef.v3n5p234.

Shamosh, N. A, \& Gray, J. R. (2008). Delay discounting and intelligence: a meta-analysis. Intelligence, 36(4), p. 289-305.

Sutter, M., Kocher, M. G., Glätzle-Rützler, D., \& Trautmann, S. T. (2013). Impatience and uncertainty: Experimental decisions predict adolescents' field behavior. American Economic Review, 103(1), p. 510-31. DOI: https://doi. org/10.1016/j.euroecorev.2018.09.004.

Tversky, A., \& Kahneman, D. (1974). Judgment under uncertainty: Heuristics and biases. Science, 185(4157), p. 1124-1131.

Tversky, A., \& Kahneman, D. (1979). Prospect theory: An analysis of decision under risk. Econometrica, 47(2), p. 263-291. DOI: https://doi.org/0012-9682(197903)47:2\%3C263:PTAAOD\%3E2.0.CO;2-3.

Weller, J. A., \& Tikir, A. (2011). Predicting domain-specific risk taking with the HEXACO personality structure. Journal of Behavioral Decision Making, 24(2), p. 180-201. DOI: https://doi.org/10.1002/bdm.677.

Wong, A., \& Carducci, B. (2016). Do sensation seeking, control orientation, ambiguity, and dishonesty traits affect financial risk tolerance? Managerial Finance, 42(1), p. 34-41. DOI: https://doi.org/10.1108/MF-09-20150256 .

Xu, Y., Beller, A. H., Roberts, B. W., \& Brown, J. R. (2015). Personality and young adult financial distress. Journal of Economic Psychology, 51, p. 90-100. DOI: https://doi.org/10.1016/j.joep.2015.08.010.

\section{Como citar este artigo}

Martelo, J. R.; Favero, E.; \& Souza Junior, W. D. de. (2020). Personalidade e decisão financeira: um estudo com jovens do Exército Brasileiro. Revista de Contabilidade e Organizações, 14:e166547. DOI: http:// dx.doi.org/10.11606/issn.1982-6486.rco.2020.166547 